\title{
Autophagy in aging and in neurodegenerative disorders
}

\author{
Yogendra S. Rajawat, Ioannis Bossis
}

University of Maryland, Department of Veterinary Medicine, College Park, Maryland, U.S.A.

\begin{abstract}
Autophagy (ATG) is the process of bulk degradation and recycling of long-lived proteins, macromolecular aggregates, and damaged intracellular organelles. Cellular homeostasis requires continuous removal of worn-out components and replacement with newly synthesized ones. Studies in yeast and other mammalian systems have increased our knowledge of the molecular mechanism of autophagy and the role of autophagy in various pathological conditions. Discovery of the genes involved in the process of autophagy has provided insight into the involvement of various molecular pathways. Growing evidence has indicated that diminished autophagic activity may play a pivotal role in the aging process. Cellular aging is characterized by a progressive accumulation of nonfunctional cellular components owing to oxidative damage and a decline in turnover rate and housekeeping mechanisms. Lysosomes are key organelles in the aging process due to their involvement in both macroautophagy and other housekeeping mechanisms. Autophagosomes themselves have limited degrading capacity and rely on fusion with lysosomes. Accumulation of defective mitochondria also appears to be critical in the progression of aging. Inefficient removal of nonfunctional mitochondria by lysosomes constitutes a major issue in the aging process. Autophagy has been associated with a growing number of pathological conditions, including cancer, myopathies, and neurodegenerative disorders. In this review, we discuss the cellular and molecular mechanisms involved in autophagy, the mechanisms of aging, and the possible role of autophagy in this process. Understanding the mechanisms by which autophagy impacts aging may provide useful molecular targets for pharmaceuticals designed to delay aging or correct conditions of premature aging.
\end{abstract}

Key words: Aging, Alzheimer's disease, Autophagy, Lifespan, Lipofuscin, Lysosomes, Mitochondria, Neurodegenerative diseases, Neuronal aging, ROS

\section{INTRODUCTION}

Homeostasis in living cells requires continuous removal of damaged and worn-out components and

Address for correspondence:

Dr. Ioannis Bossis, 8075 Greenmead Drive, Avrum Gudelsky

Building, University of Maryland, College Park, MD 20742,

Tel.: +301-314-8042, Fax: +301-314-6855,

e-mail: bossisi@umd.edu

Received 01-10-07, Revised 10-11-07, Accepted 20-11-07 replacement by newly synthesized ones. ${ }^{1}$ Turnover of all cellular components is accomplished by different degradative and synthetic pathways, which are usually complementary to each other. Different types of proteolytic systems are present in the intracellular environment that replace or repair the damaged proteins. ${ }^{2}$ These systems ensure that sufficient amounts of protein exist for the proper functioning of cells. Short-lived nuclear and cytoplasmic proteins 
are degraded by multicatalytic proteinase complexes (proteasomes) $^{3}$ and by calcium-dependent neutral proteases (calpains). ${ }^{4}$ More than $90 \%$ of cellular proteins are long-lived. Turnover of most long-lived proteins, macromolecules, biological membranes, and whole organelles, including mitochondria, ribosomes, endoplasmic reticulum, and peroxisomes, is mediated by autophagy. ${ }^{5}$ Unlike the proteasome, autophagy not only degrades proteins but can also eliminate unwanted, damaged, or redundant cell organelles or structures. Mitochondria can autonomously turn over certain defective proteins, because they possess their own proteolytic systems. ${ }^{6,7}$ Therefore, the autophagic machinery can mediate major intracellular housekeeping tasks.

Autophagy (derived from the Greek meaning "to eat oneself") is present in all eukaryotic cells and is evolutionarily conserved from yeast to humans. ${ }^{8}$ Bulk proteins and organelles are degraded by autophagy and the degradation products are recycled for the synthesis of new molecules. ${ }^{9}$ In the process of autophagy, defective and worn-out structures are sequestered into double-membrane vesicles, which are called autophagosomes. These structures are transported to and fuse with lysosomes (forming the autophagolysosome) for proteolytic degradation. The process of autophagy begins with cargo selection. After selection, the material to be degraded is engulfed in the double-membrane vesicles. Microtubule-associated protein Light Chain 3 (LC3) is recruited on the surface of the double-membrane vesicles, which subsequently surround proteins and organelles that are to be degraded. For that reason, LC3 is widely used as a marker for visualizing autophagosomes. ${ }^{10}$ Autophagosome-lysosome fusion is a multi-step process. The autophagolysomes are very short-lived structures and no reliable markers exist to visualize them (Figure 1). After being transported into the vicinity of lysosomes, the outer membrane of the autophagosome fuses with the lysosomal limiting membrane. The contents of the autophagosome are released into the lysosomal cavity, where degradation takes place by the action of hydrolytic enzymes, which is facilitated by the acidic lysosomal environment. ${ }^{11}$

\section{Forms of autophagy}

Eukaryotic cells are equipped with several deg-

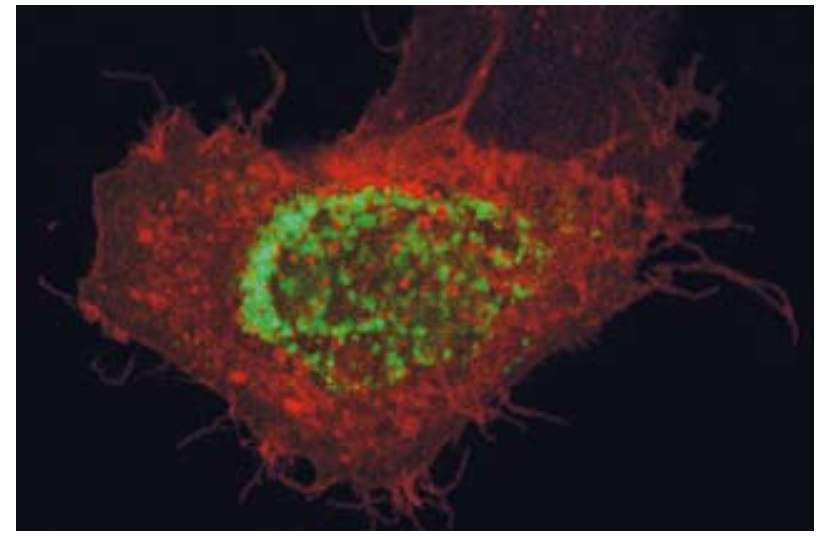

Figure 1. Autophagosmes and lysosomes. LC3 (green), a marker of mature autophagosomes, is not localized on CD63 (red) labeled organelles. CD63 primarily resides on lysosomes and plasma membrane. As yet, no reliable markers exist to monitor the transition to autophagolysosomes.

radation systems, the predominant system being the process of autophagy. Autophagy is responsible for the degradation of whole organisms and structures (bacteria, viruses), whole organelles, as well as longlived soluble proteins. ${ }^{12,13}$ Three forms of autophagic processes are currently known: classic autophagy (also called macroautophagy to distinguish it from other processes), chaperone-mediated autophagy, and microautophagy. ${ }^{14}$

Classic autophagy is an inducible form of autophagy that becomes activated under stress conditions (nutrient starvation, infections, and toxins). ${ }^{15}$ Most eukaryotic cells are equipped with another form of autophagy, known as Chaperone-Mediated Autophagy (CMA). ${ }^{16}$

CMA is selective for a particular group of soluble cytosolic proteins that contain a specific sequence signature. Unlike autophagy, CMA does not require intermediate vesicle formation. ${ }^{17}$ Once the specific signature sequence is identified, these soluble proteins are directly translocated to the lysosomal membrane. The specific signature sequence, which is present in all CMA substrates, is biochemically related to the pentapeptide KFERQ ${ }^{18}$ All substrates with this sequence are then targeted to lysosomes. The cytosolic molecular chaperone heat shock protein 70 (hsp70) and its co-chaperones recognize the signature motif in substrate proteins. ${ }^{19}$ The substrate/chaperone complex is then targeted to the lysosomal membrane, where it 
binds to a receptor protein, the Lysosome-Associated Membrane Protein type 2a (LAMP-2a) ${ }^{20}$ A second chaperone, lysosomal hsc73 (lys-hsc70), is required for complete translocation of the substrate protein/ hsc70 complex into the lysosomal matrix, where it is completely degraded by lysosomal proteases. ${ }^{21}$

The third form of autophagy is microautophagy. ${ }^{14}$ The characteristic feature of the microautophagy process is that the lysosomal membrane itself either invaginates or exvaginates to engulf an organelle. Unlike macroautophagy, there is no sequestering doublemembrane formed in the cytoplasm. ${ }^{22}$ Pexophagy $^{23}$ and Mitophagy ${ }^{24}$ involve the selective sequestration or engulfment of peroxisomes and mitochondria, respectively, and their delivery to lysosomes.

There is one more pathway, found in the yeast $S$. Cerevisiae, which is closely related to the process of autophagy, called cytoplasm-to-vacuole targeting (Cvt) pathway. ${ }^{25}$ The major function of this pathway, which plays a pivotal role in the biosynthetic and physiological role of yeast cells, is to deliver the enzymes aminopeptidase I and alpha-mannosidase to the yeast vacuole. ${ }^{26}$ Several genes that are involved in the Cvt pathway are also involved in the classic autophagy pathway. ${ }^{27}$

\section{The autophagy process}

The stages involved in the process of autophagy have not as yet been precisely delineated, especially for mammalian cells. Studies in the yeast $S$. Cerevisiae have provided insight into the mechanisms involved and, based on these studies, the following steps of ATG are largely accepted. ${ }^{28}$

\section{Induction and cargo selection}

Autophagy may be either a selective or nonselective process. ${ }^{29}$ In S. Cerevisiae, Cvt is a transport mechanism involved in the recognition and packaging of cargo and is considered a selective process. However, this pathway is limited only to yeast; it is not present in any other organism. ${ }^{30}$ At present, autophagy is viewed as a nonselective process, where cytoplasmic structures and organelles are randomly enwrapped into double-membraned structures. ${ }^{31}$ Nonetheless, the possible presence of a mechanism like Cvt in mammalian cells cannot be excluded. At the molecular level (Figure 2), the serine/threonine protein kinase
TOR (Target Of Rapamycin) is involved in the induction of autophagy. ${ }^{31}$ Activated TOR (phosphorylated form) is part of the induction complex and acts as a negative regulator of autophagy..$^{32}$ Phosphorylated Tor negatively controls autophagy, primarily by acting on the signaling cascade that controls general translation and transcription. In addition, activated Tor induces hyperphosphorylation of Atg13, which lowers its binding affinity to other Atg interacting proteins, thereby inhibiting autophagy. ${ }^{33}$

\section{Vesicle nucleation}

The initial formation of an autophagosomal membrane takes place by enwrapping the degradative cargo within a double membrane, which eventually elongates to form a vesicle called an autophagosome. The exact origin of autophagosomal membranes is controversial. Studies in mammalian cells have suggested that autophagosomal membranes originate from the ribosome-free region of the rough endoplasmic reticulum..$^{34}$ Alternatively, a poorly-characterized organelle, called a phagophore, has also been suggested as the origin of autophagosomes and other vesicular structures. ${ }^{35}$ In yeast, a unique perivacuolar structure, called PAS (preautophagosomal structure), has been proposed as the precursor of autophagosomes due to the transient association of several Atg proteins with it. ${ }^{36}$ A PAS-like structure has not yet been identified in any other endomembrane system. ${ }^{37}$

\section{Vesicle expansion and retrieval}

Vesicles will elongate and extremities will fuse to complete the double-membraned structure. Phagophores elongate and completely encircle the cargo to form an 'autophagosome' or 'autophagic vacuole'. ${ }^{12}$ Most of the proteins involved in vesicle expansion and maturation steps are retrieved to the original pool because they do not associate with the complete and mature autophagosome. ${ }^{8,12,14}$ Therefore, it has been suggested that the proteins involved in autophagosome formation are retrieved for future use. Atg8 (LC3) is the exception; it is found on the mature autophagosome and can be used as a valuable marker to track these structures ${ }^{38}$ (Figure 1).

Vesicle targeting, docking, and fusion with lysosomes

The next critical step is transport and fusion of the 
autophagosome with the lysosomes. The outer membrane of the autophagosome completely fuses with the outer membrane of the lysosome to make a path for the inner membrane bound autophagic vacuole. The inner membrane and its enclosed cytoplasmic contents, which together are called an 'autophagic body', are released into the lumen of the lysosome. The machinery required for the process of vesicle fusion includes SNARE proteins and the class C Vps/HOPS complex. ${ }^{13}$

\section{Vesicle breakdown and recycling}

The ultimate step in the autophagic process is degradation of the autophagic body content by lysosomal enzymes, which ensures recycling of essential cytoplasmic contents.

\section{Molecular machinery involved in autophagy}

Various extracellular and intracellular stimuli can induce and modulate autophagy, including nutrient regulation, cell proliferation, cell size regulation, differentiation, apoptosis, hormone or therapeutic treatment, accumulation of misfolded proteins and damaged organelles, and invasion of microorganisms. Discovery of the $A T G$ genes in yeast has greatly advanced our understanding of the molecular mechanisms involved in autophagosome biogenesis and the various pathways that regulate autophagic activity. ${ }^{39}$ Most yeast $A T G$ genes have mammalian homologues, which suggests that the basic machinery for autophagy has been evolutionarily conserved in eukaryotic organisms. These genes, also called APG/AUT/CVT, were initially identified from studies that investigated defects in the formation of autophagic vacuoles and protein degradation in S. Cerevisiae. ${ }^{40,41}$ In these studies, knockout strains of individual ATG genes showed defects of starvation-induced autophagy. It is now widely accepted that at least 28 yeast genes are actively involved in autophagy. More than 50 additional yeast genes may be required for autophagy, which also play key roles in other pathways. ${ }^{42}$ The precise cellular and biochemical function of these genes in the autophagic pathway remains to be elucidated.

Regulation of autophagosome biogenesis, maturation, and fusion with lysosomes is a complicated process, a number of diverse signaling complexes and pathways being involved (Figure 2). Many signaling pathways, including Tor or mammalian Target Of Rapamycin (mTOR), Phosphatidylinositol 3-Kinase-I (PI3K-I)/PKB, Class III PI3K, Akt-PTENTSC1/2-Rheb-mTOR circuit, the Insulin Receptor and downstream targets, GTPases, calcium, and protein synthesis all play important roles in regulating autophagy ${ }^{43}$ In general, Class I PI3K, Akt, Rheb and mTOR components inhibit autophagy, while Class III PI3K, PTEN and TSC1 and 2 positively regulate autophagy. Cross-talk between these regulatory pathways mediates the response of cells to various challenges that affect autophagy. ${ }^{44}$

The Tor signaling pathway, the Atg1 complex, and the Vps34/class III PI3K complex are critical in initiating an autophagic response. TOR is a classic serine/threonine kinase. Nutrient starvation or treatment of yeast cells with rapamycin (a specific inhibitor of TOR) rapidly induces an increase in autophagosome biogenesis and autophagic activity. ${ }^{45}$ Inhibition of Tor results in dephosphorylation of Tap, ${ }^{42}$ causing its dissociation from PP2A. PP2A becomes activated, causing dephosphorylation of downstream targets, which leads to a variety of antiproliferative responses and induction of autophagy. In mammalian cells, mTOR appears to modulate autophagy in a manner similar to that observed in yeast. Likewise, the activity of the mammalian PP2A orthologue is strongly correlated to induction of autophagy. ${ }^{46}$ Recently, association of protein kinase A components with mTOR in regulation of autophagy has been observed. Depletion of the type IA regulatory subunit (RIalpha) of Protein Kinase A (PKA) in mammalian cells and tissues activates mTOR and causes autophagic deficiency. ${ }^{47}$ Further, it has been shown that mTOR and the regulatory subunit of protein kinase A (PRKAR1A) spatially and functionally interact during autophagosome maturation. ${ }^{48}$ However, the response of mammalian cells to rapamycin is more diverse; certain cell types respond but others do not.

A second autophagy signaling complex includes Atg1, a protein kinase involved in both yeast Cvt and autophagic pathways, Atg11, and Atg13. ${ }^{49}$ Under nutrient-rich conditions, Tor activation causes hyperphosphorylation of Atg13 that prevents its association with Atg1, which subsequently enables interaction of Atg1 with Atg11; this may determine the use of the 


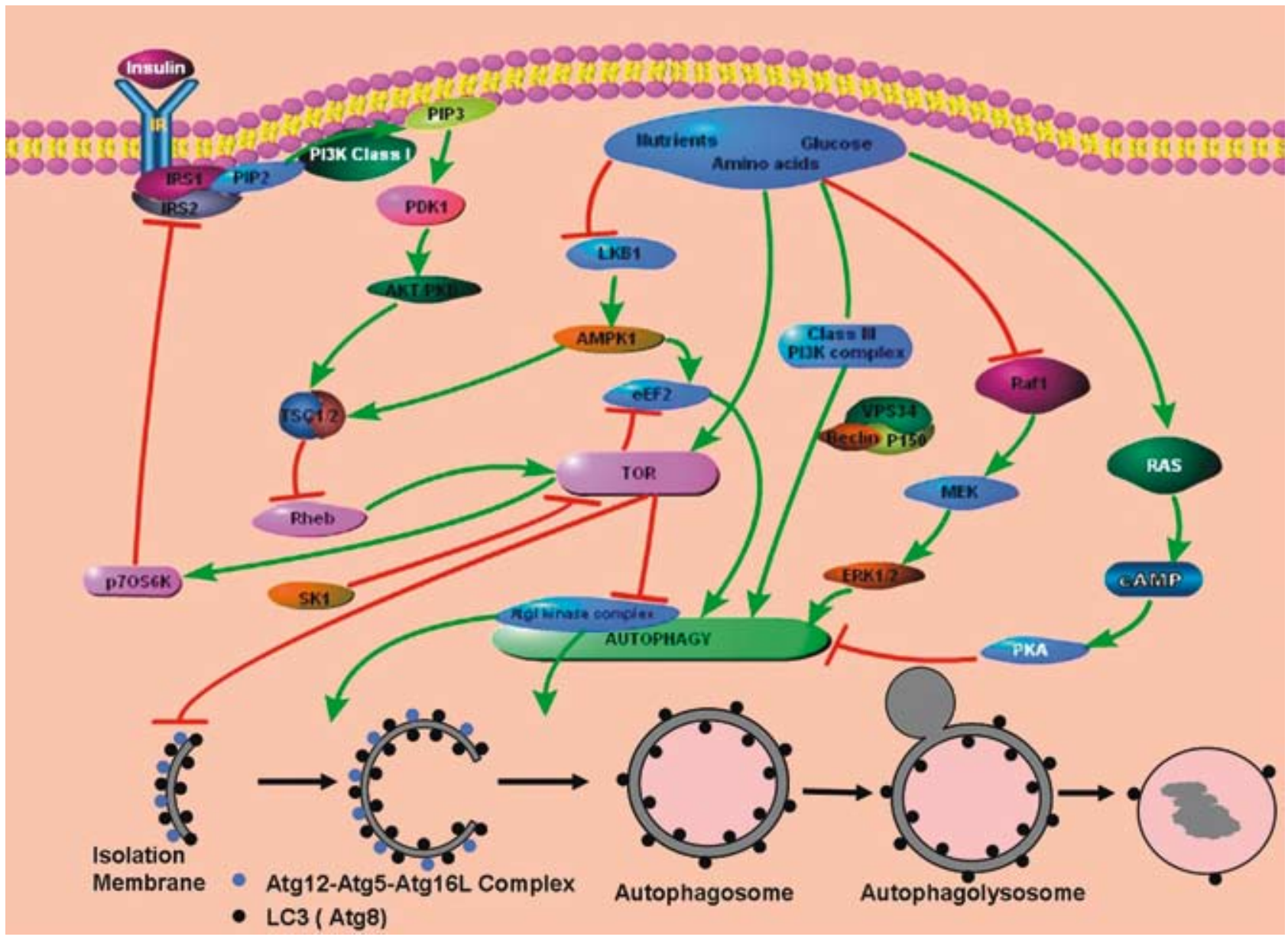

Figure 2. Pathways Regulating Autophagy: TOR (Target Of Rapamycin) is at the center of the diverse pathways linked to autophagy. The PI3K signaling pathway has been extensively characterized and involves the class I and class II PI3Ks. The class I PI3 kinases link autophagy to cancer through the AKT/PKB pathway. The class II PI3K pathway, which is mediated by beclin1, also plays an important role in regulation. MAPKinase pathway is also involved in regulation of autophagy through Raf- 1 and Ras.

autophagic machinery in the Cvt pathway. Under nutrient starvation or treatment with rapamycin, Atg13 becomes partially dephosphorylated, leading to an Atg1-Atg13 interaction, which subsequently triggers autophagy and generation of autophagosomes instead of Cvt vesicles. In mammalian cells, GATE-16 (Golgi associated ATPase enhancer of 16 $\mathrm{kDa})$ and GABARAP ( $\gamma$-aminobutyric acid type A receptor-associated protein $)^{50}$ were found to interact with the ULK1 kinase. Since GATE-16, GABARAP, and MAP-LC3 (microtubule-associated protein light chain 3) are putative orthologues of yeast Atg8a (essential for autophagosome formation), it has been postulated that ULK1 may be the mammalian orthologue of Atg1..$^{51}$
The third major autophagy signaling pathway involves the Vps34/class III Phosphatidylinositol 3-Kinase (PI3K) complex. The observation that 3methyladenine (PI3K inhibitor) inhibits autophagy was the first evidence that implicated this family of kinases in the process. ${ }^{52}$ In addition, other PI3K inhibitors, such as wortmannin and LY294002, have also been found to inhibit autophagy. In yeast, Vps34 is primarily involved in vacuolar protein targeting, through the endosomal/prevacuolar compartment, to form a complex with Vps15-Vps38-Atg6..$^{53}$ At the same time, $\mathrm{Vps} 34$ has been found to associate with Vps15-Atg6-Atg14 on pre-autophagosomal membranes. However, the mechanism by which Vps34 affects autophagosome formation is largely unknown. 
In mammalian cells, there are three classes of PI3Ks. So far, only Class I and Class III PI3K have been implicated in autophagy, primarily in the early steps of autophagosome formation. ${ }^{54}$ Class III PI3K has been found to associate with beclin-1 and p150, which are the orthologues of yeast Atg6 and Vps15, respectively. Class III PI3K regulates diverse molecular pathways, including several involved in tumor formation. The first autophagy-related tumor suppressor gene reported was Beclin-1, the mammalian orthologue of yeast Atg6, which showed a relationship between autophagy and cancer. ${ }^{55}$

\section{Aging}

Aging is an essential, inevitable physiological phenomenon characterized by the accumulation of deleterious changes in cells and tissues during the post-maturational deterioration, which decreases the ability to survive and increases risk of death. ${ }^{56}$ Aging changes are the result of predetermined genetic factors, environmental influences, and certain diseases. Aging is grossly characterized by an accumulation of worn-out organelles and various cellular substructures over time. These progressive accumulations reduce cellular efficiency in various biological processes that are required to maintain homeostasis and survival. Malfunctions in the biological processes required for the maintenance, repair, and turnover pathways may be the main cause of the cumulative cellular damages during aging. ${ }^{57}$ The balance between synthetic and catalytic pathways controls the turnover rate of cellular components, which in turn depends on the physiological condition of the cells.

A general consensus on the fundamental mechanism of aging exists. During the lifespan of an organism, cells are subjected to various destructive forces, which may originate from either internal or external sources. The result of continuous exposure to these harmful forces is progressive accumulation of lesions. Over time, these lesions become detrimental to cell and tissue survival. The precise molecular mechanism of aging is not yet completely understood, but the elements that are responsible for oxidative damage and improper housekeeping are considered the main contributory causes. These two factors are considered to play pivotal roles in cell survival. ${ }^{58}$

We cannot point out a single factor responsible for the process of aging, due to the complexity of the whole process. For proper functioning, adaptation, and survival in harsh environments, various cells have to function in unity. These types of cells have diverse kinds of machinery which accomplish their assigned functions and several pathways are involved. Aging is thus seen to be a complex, interwoven, multi-factorial process that depends on the cross-talk between different cells and different molecular pathways within the cells. Several theories of the aging process have been proposed, the fundamental concept behind these theories being the build-up of "damage" that occurs throughout the entire lifespan of cells. Such damage may accumulate from toxic byproducts of routine metabolism or inefficient repair/defensive systems. It is apparent that in long-lived cells, such as neurons or cardiomyocytes, accumulation of lesions can be more detrimental. In proliferating cells, lesions rarely accumulate with age while the process of cell division seems to efficiently remove damaged structures. ${ }^{59}$

Current theories of aging cannot in themselves completely explain the entire process; however, each one of them can partially contribute to the overall understanding of the phenomenon. Notwithstanding that these various theories cannot as yet be clearly demarcated, they have been categorized as evolutionary, molecular, cellular, and systematic. ${ }^{60}$

\section{Theories of aging}

First proposed in $1956,{ }^{61}$ the "oxidative damage" theory of aging postulates that an accumulation of oxidized macromolecules causes a progressive functional deterioration of cells, tissues, and organ systems, which subsequently may lead to cell death. Reactive Oxygen Species (ROS) are generated in the normal oxygen metabolism of cells. If not neutralized or eliminated, these harmful free radicals may cause deleterious effects, such as DNA-strand breaks, DNA-protein crosslinking, protein fragmentation, and lipid oxidation, which in turn results in formation of hydroperoxides, alkyl radicals, and aldehydes. Normally, cells have their own protective mechanisms (superoxide dismutase, catalase, glutathione peroxidase, vitamine E) whose function is to reduce the formation of free radicals. However, total elimination cannot be accomplished. Basically, the oxidative damage theory proposes that 
ROS formation is inherent in biological systems and causes cumulative damage and senescence. ${ }^{62}$

The oxidative damage theory can be further categorized based on the individual role of certain organelles in the aging process. The mitochondrial theory of aging proposes that it is the accumulation of damage to mitochondria and mitochondrial DNA (mtDNA) that leads to aging in humans and animals. ${ }^{63,73,74}$ Mitochondria, the powerhouse of cells, produce the energy (in form of ATP) required for all cellular functions. During the process of energy production, mitochondria also produce superoxide ions, hydrogen peroxide, hydroxyl radicals, and ironcentered free radicals. ${ }^{64}$ These free radicals, as well as ROS, produced inside the mitochondria via the Electron Transfer Chain (ETC), make them prone to damages, both at the level of protein and mtDNA. In addition, mtDNA has properties that make it susceptible to deleterious mutations. mtDNA lacks introns and has no efficient DNA repair mechanisms. These characteristics increase the possibility that random mutations cannot be repaired and that they are more deleterious. ${ }^{65}$ Another phenomenon frequently observed in aging cells is the accumulation of mitochondria with mutated DNA. Often, complete replacement of normal mitochondria with mutated mitochondria is observed (homoplasmy). ${ }^{66}$ It has been proposed that mutated $\mathrm{mtDNA}$ may have a replicative advantage over normal mtDNA; consequently, there will be increased replication (clonal expansion) of defective mitochondria. ${ }^{65}$ Occurrence of homoplasmic mtDNA mutations in normal intestinal epithelial, ${ }^{67}$ as well as in neoplastic cells ${ }^{68}$ provides support for this concept. Development of premature aging in different organs of homozygous mtDNA polymerase gamma knockout mice provides further support for the role of mitochondrial damage in aging. ${ }^{69}$

Despite the presence of various protective proteases (membrane-bound $\mathrm{AAA}^{6}$ and matrix Lonproteases $\left.^{7}\right)$, mitochondria are unable to effectively degrade damaged proteins. During aging, the change in morphology and function of mitochondria is remarkable. Mitochondria become excessively enlarged (in this form usually referred to as giant mitochondria) and exhibit complete loss of cristae and destruction of inner and outer membranes. These altered mitochondria cannot perform ETC or respiratory chain func- tions, a phenomenon which results in the formation of amorphous electron-dense material. ${ }^{70}$ Autophagy is the protective mechanism used by cells to remove damaged mitochondria and mutated mtDNA. ${ }^{71}$ In younger organisms, most defective mitochondria are removed by autophagy. As mitochondrial damage progressively increases with age, the accumulation of defective and enlarged mitochondria becomes more prominent. Recent experiments have indicated that small mitochondria are degraded more efficiently by autophagy than larger ones..$^{70}$ The decline in autophagic activity with age plus the inability to efficiently remove large mitochondria provide an explanation for the apparent accumulation of giant mitochondria in postmitotic aging cells. ${ }^{72}$

Significant changes have been observed in peroxisomes during aging and it is has been hypothesized that peroxisomes may be involved in the process of aging. ${ }^{75}$ Increased generation of peroxisomal ROS and alterations in fatty acid oxidation activity are the two major observations that implicate peroxisomes in the aging process. Changes in fatty acid oxidation may lead to altered membrane lipid composition. Several investigators have suggested the essentiality of various membranes in maintaining intracellular homeostasis and that deterioration of membrane integrity is the underlying cause of the aging process. ${ }^{76}$ Many age-related membrane alterations have been reported, including lipid peroxidation, ${ }^{77}$ as well as accumulation of the lipid dolichol, which can greatly impair transmembrane signaling. ${ }^{78}$ Interestingly, the degree of dolichol accumulation in the liver of growing rats is highly correlated with levels of autophagic activity (both macroautophagy and pexophagy) in the same organ. ${ }^{79}$

The glycation/cross-linking hypothesis of aging is based on observations that our proteins, DNA, and other structural molecules develop cross-links to one another with age.$^{80}$ The main way cross-linking occurs is through a process called glycation. Postranslationally processed proteins contain sugar moieties. These glycated molecules can be reduced through a complex series of reactions (Maillard reaction) to yield a multitude of end-products known as Advanced Glycation End-products (AGEs). When both of the sticky ends of AGEs adhere to neighboring proteins, they form permanent, disabling cross-links. Damaged proteins 
are normally broken down by proteases; however, in the presence of cross-linkages, proteases are inhibited and, as a result, damaged proteins accumulate. ${ }^{81}$

Several studies have strongly suggested involvement of glycation/cross-linking in aging. Cross-linking of collagen has been shown to be at least partially responsible for some age-related changes in skin. ${ }^{82}$ Cross-linking of proteins in the lenses of the eyes plays a significant role in age-related cataract formation. ${ }^{83}$ Cross-linking of proteins in arterial walls may account for at least some forms of atherosclerosis, like diabetic angiopathy ${ }^{84}$ as well as age-related decline in kidney function. Recent evidence has also indicated that glycation contributes to the formation of beta-amyloid, the protein that clumps together in the brains of Alzheimer's patients.

\section{LYSOSOMAL FUNCTION DURING AGING}

Lysosomes are the major organelles responsible for proteolytic degradation and recycling. The term lysosome derives from the Greek words lysis (dissolution or destruction) and soma (body). After the discovery of lysosomes by the Belgian cytologist Christian de Duve in $1959,{ }^{85}$ lysosomes have been described as the cellular demolition crew or garbage disposal system. They are frequently nicknamed "suicide-bags" or "suicide-sacs" by cell biologists due to their role in autolysis. The main function of these microscopic organelles is the digestion of macromolecules derived from phagocytosis, endocytosis, and autophagy (Figure 2). As mentioned earlier, autophagy is a catabolic process that eliminates aggregates of aberrant proteins, superfluous or damaged organelles, and sometimes even entire organisms such as invading bacteria. Although autophagosomes possess some hydrolytic activity, this activity is insufficient to complete proteolytic degradation. Thus, fusion of autophagosomes with lysosomes can be viewed as the Achilles' heel in the process of autophagy.

There are various pathways by which lysosomes receive the extracellular and intracellular material that is to be degraded.$^{86}$ Endocytosis can internalize extracellular proteins, as well as integral membrane proteins, and sequester them in endosomes. Subsequently, these endosomes fuse with lysosomes and deliver the cargo. Crinophagy is the process by which secretory proteins are delivered to lysosomes by fusion with secretory vesicles. Autophagy is the bulk degradation of cytoplasm and organelles by promoting the transfer of material from one, topologically distinct compartment, to another-from the cytosol to the vacuole in yeast, or to lysosomes in eukaryotic cells. Chaperone-Mediated Autophagy (CMA) is a more selective degradation process of autophagy in which a specific sequence in the target substrate protein is recognized by cytoplasmic molecular chaperones. Subsequently, the substrate-chaperone complex is delivered to lysosome for degradation.

The activity of lysosomes is pivotal in aging cells. Age-related decline in overall proteolytic activity has been observed in almost all organisms that have been studied, and specific age-related defects in the different proteolytic systems have been reported ${ }^{87}$ Reduced proteolysis with aging is most evident for long-lived proteins, some of which are known substrates for lysosomal pathways of degradation. Non-lysosomal proteolytic pathways either show very little change (ubiquitin-proteasome pathway) ${ }^{88}$ or increase (calpains $)^{89}$ with age. Therefore, the lysosomal pathway has become the focus of investigations in pursuit of likely explanations for reduced proteolysis with age.

Age-related alterations in the lysosomal system are clearly noticeable in the form of a pigmented product (lipofuscin) that accumulates in lysosomerelated vesicles as a result of incomplete digestion of engulfed components. The lysosomal compartment is rich in iron molecules. Diffusion of cytoplasmically produced peroxides into the lysosome could result in Fenton-type reactions and hydroxyl radical production, which in turn can lead to peroxidation of the lysosomal protein content and formation of lipofuscin. Lipofuscin is a yellowish-brown, autofluorescent, non-degradable polymeric substance. ${ }^{90}$ Accumulation of lipofuscin reduces the degradative capacity of lysosomes, ${ }^{91}$ which in turn results in further accumulation of waste material inside the lysosome. The rate of lipofuscin formation is inversely related to age, and lysosomes filled with lipofuscin have a reduced ability to fuse with autophagic structures..$^{92}$ The molecular basis of the age-related decline in different autophagic pathways is the subject of intense investigation. ${ }^{8}$ 
As mentioned earlier, lipofuscin is non-degradable and cells cannot get rid of it. ${ }^{93}$ However, actively dividing and growing cells can partially eliminate lipofuscin by diluting the pigment in each mitotic cycle. Post-mitotic cells do not have this capability. It has previously been observed that while accumulation of lipofuscin in postmitotic cells of short-lived animals is very rapid, in long-lived animals it is very slow. ${ }^{94}$ This finding suggests a relationship between lipofuscin accumulation, function of the lysosomal compartment, and cell survival. It is also worth mentioning that mitochondria of long-lived animals tend to produce significantly less superoxide and hydrogen peroxide than short-lived ones. ${ }^{95}$

The presence of undigested materials in lysosomes could be responsible for their impaired ability to fuse and/or degrade the autophagosome contents. A decrease in the lysosomal levels of the CMA receptor (LAMP-2A) is the primary defect responsible for the diminished CMA activity during aging. ${ }^{92}$ Normal CMA activity is initially maintained (during middle age) by increasing the amount of luminal chaperone. At advanced ages, the levels of the receptor are so low that compensation by the chaperone is no longer possible..$^{96}$ The reasons behind the decline in receptor numbers are not known and are currently under investigation.

\section{Role of autophagy in diseases}

Several human diseases are associated with decreased autophagic activity, particularly in nondividing cells of the nervous and muscle systems where turnover of intracellular proteins may be critical. Muscular disorders, known as vacuolar myopathies, are associated with massive accumulation of imperfect autophagic or lysosomal vacuoles. ${ }^{97}$ Danon disease is characterized by cardiomyopathy and mild mental retardation and is caused by a deficiency in LAMP-2, the transmembrane protein involved in endosomal and lysosomal fusion during the late maturation phase of the autophagosome. ${ }^{98}$ Accumulation of aberrant autophagosomes has also been associated with X-linked myopathy (X-MEA) ${ }^{99}$ inclusion body myositis, ${ }^{100}$ and Marinesco-Sjögren syndrome. ${ }^{101}$

Reduced autophagic activity is also associated with neurodegenerative disorders, such as Parkinson's (PD), ${ }^{102}$ Alzheimer's (AD), ${ }^{103}$ Huntington's (HD), ${ }^{104}$ and transmissible spongiform encephalopathy (prion disease) ${ }^{105}$ Altered activity of proteolytic systems and the occurrence of intracellular protein aggregates (inclusion bodies) are characteristic of these neurodegenerative diseases. The protein that accumulates in PD is mutated $\alpha$-synuclein, ${ }^{106}$ in HD the protein is an abnormally expanded form of huntingtin, ${ }^{107}$ and in $\mathrm{AD}$, protein deposits of overproduced $\beta$-amyloid precursors. Reduced autophagy has also been associated with chronic liver disease and hepatocellular carcinoma due to retention of mutant alpha (1)-AntiTrypsin Z (ATZ) protein in the ER and mitochondria of hepatocytes. ${ }^{108}$ One could hypothesize that perturbations at any stage of autophagosome biogenesis, maturation, or fusion with lysosomes would result in progressive accumulation of these aggregates and lead to disease progression.

In addition to the classic aging disorders and those of premature aging, autophagy has also been implicated in pathogenesis of infectious diseases. In general, autophagy functions as a protective mechanism against infections. Most foreign invaders (bacteria, parasites, viruses) must actually bypass the autophagic line of defense before they can establish infections. In certain cases, some microorganisms have developed strategies to manipulate the autophagic machinery to their own advantage. After escaping the endophagocytic pathway and preventing fusion with lysosomes, the bacterium Brucella abortus shelters and replicates itself in autophagosome-like vesicles. ${ }^{109}$ Legionella pneumophila secretes a product that activates autophagy and allows it to replicate itself directly inside autolysosomes. ${ }^{110}$ Likewise, a virulence protein, ICP34.5, in the Herpes simplex virus antagonizes the autophagic pathway and allows the virus to escape degradation. ${ }^{111}$ Recent immunological studies have also indicated the importance of autophagy in antigen presentation and successful cellular defense. It can be speculated that the decline in autophagic activity with age may contribute to the apparent increased risk of infectious disease in the elderly.

Since autophagic elimination of aggregated proteins and damaged organelles preserves cells from further damage, autophagy could serve a protective role. Enhancement of autophagic activity in aging organisms can have preventive or therapeutic potential. Caloric restriction-induced autophagy has a 
well-documented effect in extending life expectancy, at least in lower eukaryotic organisms and some laboratory mammals. ${ }^{112}$ The mTOR inhibitor and autophagy promoter, rapamycin, has shown promising anticancer activity in clinical trials. ${ }^{113}$ Bacterial and viral proteins that modify autophagosome formation could also be harnessed to modulate this degradative pathway for therapeutic purposes.

\section{AUTOPHAGY AND NEURONAL AGING}

Autophagy has primarily been implicated in various neurodegenerative disorders because of the apparent accumulation of aberrant structures and macromolecules in neuronal cells. Since neurons have very limited capabilities for cell replication and renewal, one would anticipate that any perturbations in autophagic activity would first affect the neuronal tissues. Autophagy dysfunction is emerging as a theme in neurodegenerative diseases in which mis-aggregated proteins accumulate. It has long been puzzling why protein aggregation and neurotoxicity develop so late in life, even in familial forms of diseases where the mutant protein is present throughout life. It now seems likely that declining efficiency of protein turnover is partly responsible. Both macroautophagy and CMA slow considerably during normal aging, contributing directly in the declining of tissue performance. ${ }^{87}$ Genetic ablation of autophagy in mice has recently been shown to induce neurodegeneration and accumulations of ubiquitinated proteins. ${ }^{114}$ Moreover, some gene mutations that cause neurodegenerative diseases directly impair the proteolytic systems responsible for degrading the mutant protein.

\section{I) Parkinson's disease}

Parkinson Disease (PD) is characterized by progressive death of dopaminergic neurons in the substantia nigra. Accumulation of $\alpha$-synuclein, a cytosolic protein with synaptic functions, takes place within intracellular inclusion bodies called Lewy bodies. The presence of Lewy bodies is a hallmark feature of this disorder. Neuronal cell death in PD is more complex. In addition to the accumulation of aberrant autophagosome-like structures, there is apoptosis and necrosis. ${ }^{115} \mathrm{CMA}$ is considered the rate-limiting degradative mechanism in neuronal cells, but both macroautophagy and proteasome pathways might be involved in $\alpha$-synuclein turnover. ${ }^{114,115}$ In cases of familial PD, mutant $\alpha$-Synuclein cannot efficiently enter lysosomes because of abnormally high affinity to lysosomal receptor LAMP-2A. Due to high binding affinity for the receptor, the substrate to be degraded cannot be translocated properly. The high affinity of mutant $\alpha$-synuclein to LAMP-2A also blocks uptake of other CMA substrates and thereby prevents their degradation. ${ }^{5,117}$ Impaired CMA is usually accompanied by accumulation of abnormal autophagic vacuoles and subsequent cell death. It has been hypothesized that when macroautophagy is induced to compensate for the CMA defect, impaired autophagosome-lysosome fusion and incomplete lysosome acidification occur. ${ }^{116}$

\section{II) Alzheimer's disease}

Alzheimer's Disease (AD) is characterized mainly by gradual neuronal loss and progressive dementia. The observed cell damage is associated with intraneuronal fibrillary tangles and extracellular senile plaques. In addition to the well-documented lysosomal dysfunction in $\mathrm{AD},{ }^{119}$ significant alteration in autophagic activity has recently been indicated. ${ }^{118}$ The hallmark feature of AD pathology is the presence of enormously swollen 'dystrophic' neuritis in which autophagic vacuoles progressively accumulate and become the predominant organelle. ${ }^{118}$ In normal brain cells, autophagosomes are usually found in low numbers. However, in a large number of brain cells from $\mathrm{AD}$ patients, autophagosomes are very prominent. This probably indicates activation of autophagy but with impaired maturation and/or impaired autophagosome-lysosome fusion, ${ }^{120}$ which eventually leads to apoptosis. A key pathogenic factor in $\mathrm{AD}$ is generation of Amyloid- $\beta$ peptide $(A \beta)$ by the activity of autophagosome hydrolases. ${ }^{119} \mathrm{~A} \beta$ is the proteolytic product of a transmembrane protein that actually resides in autophagosome membranes. In the normal brain, most $\mathrm{A} \beta$ formed during normal autophagosome biogenesis is degraded within the lysosomes. In AD brains, diminished autophagosome-lysosome fusion results in accumulation of $A \beta$-filled autophagosomes, which constitute a major intracellular reservoir of the toxic peptide. ${ }^{121}$

Excessive generation of $\mathrm{A} \beta$ in $\mathrm{AD}$ brain cells is also driven by increased levels of the $\gamma$-secretase 
complex within autophagosomes. The $\gamma$-secretase complex mediates the cleavage of APP to A $\beta$ within autophagosomes. Most familial, early-onset cases of $\mathrm{AD}$ are due to mutations on the Presenilin-1 gene, a component of the $\gamma$-secretase complex. Mature autophagosome membranes are highly enriched with Presenilin-1. During the early stages of induction and autophagosome biogenesis, presenilin-1 translocates to the immature autophagosomal vesicles and is suspected of being involved in regulation of autophagosome maturation. Mutations of presenilin-1 in early-onset $\mathrm{AD}$ accentuates lysosomal pathology and promotes $\mathrm{A} \beta$ pathology and neuronal cell loss. ${ }^{122}$ In support of this possibility are observations that the turnover of several proteins is impaired in blastocysts that lack the genes encoding presenilins- 1 and -2 , leading to the increased appearance of these proteins within autophagic vacuoles. ${ }^{123}$

\section{III) Huntington's disease}

Huntington's Disease (HD) is one of the best characterized polyglutamine-expanded diseases. These diseases are caused by mutations that result in abnormally long sequences of polyglutamine residues, which make proteins aggregation-prone and toxic. Polyglutamine disorders also include spinal and bulbar atrophy, spinocerebellar ataxia, and dentatorubralpallidoluysian atrophy. ${ }^{124}$ Autophagy plays a crucial role in the pathogenesis of $\mathrm{HD}$, as polyglutamine sequences are poor substrates for the proteasome degradation pathway. Degradation of proteins that contain polyglutamine sequences is dependent largely on autophagy. ${ }^{125}$ Dependency on autophagy is evidenced by the accumulation of highly ubiquitinated aggregates of huntingtin (htt) in the endosomal-lysosomal organelles of neurons ${ }^{126}$ and lymphoblasts ${ }^{127}$ in patients with HD. When over-expressed in cells, htt accumulates in autophagic compartments, the degree of accumulation being proportional to the length of the polyglutamine sequence in the protein. ${ }^{128}$ Experimental inhibition of autophagosome biogenesis and/or fusion with lysosomes increases htt aggregation in cells both in vitro and in vivo. ${ }^{129} \mathrm{In}$ contrast, rapamycin treatment reduces htt accumulation and neurodegeneration in cell and fly models of polyglutamine disease and reduces neurological deficits and htt aggregation in a mouse model of HD. ${ }^{130}$ This strongly suggests that inadequate levels of autophagy contribute to the complex neuronal cell death pattern in this disease. ${ }^{131}$ The mechanism by which htt aggregates inhibit autophagy is not known. However, sequestration of endogenous autophagy inhibitors (mTOR) to the sites of autophagosome maturation has been speculated. ${ }^{48,132}$

\section{CONCLUSIONS AND FUTURE PROSPECTS}

Over the last four decades, our understanding of the autophagic process has increased enormously. Genetic screening approaches using yeast have addressed many questions concerning the molecular mechanisms of autophagy. Nevertheless, the interplay between the different signaling complexes involved in the process and the specific mechanisms of autophagosome biogenesis, transport, and fusion warrant further exploration. Despite recent findings that have shed some light on the major cellular and molecular events involved in mammalian autophagy, our knowledge regarding the autophagic pathway in mammalian cells remains rather limited. Discovery of a larger number of Autophagy-related genes ( $A T G$ genes) in mammals compared to those in lower eukaryotes suggests that autophagy is a much more complex process in higher organisms. Generation and analysis of transgenic and knockout animal models will be essential to understand the evolutionarily acquired complexity of autophagy mediated processes in mammals. ${ }^{133}$ Longevity studies on these animal models would also help to assess components of the aging phenotype and directly link them to autophagic malfunction.

A decrease in the autophagic activity has been convincingly demonstrated in older organisms. It has been proposed that accumulation of aberrant or functionally disabled mitochondria and other damaged structures is a consequence of an age-related decline in autophagic and lysosomal activity. ${ }^{134}$ Furthermore, it has been reported that the decline of autophagy with age is affected by alterations in glucose metabolism and hormone levels which are inherent to aging. ${ }^{135}$ These observations, together with the finding that autophagy genes are essential for lifespan extension in Caenorhabditis elegans, ${ }^{136}$ and that inhibition of Tor kinase (whose activity inhibits autophagy) doubles the lifespan of this nematode, ${ }^{137}$ support the idea that dysfunction of the autophagic 
pathway may induce premature aging.

Considerable advances have been made in our understanding of the defective steps that lead to dysfunction of autophagy during aging. However, most of the molecular components responsible for diminished autophagic activity with aging still remain elusive. Age-related research should very soon benefit from current advances in the molecular dissection of macroautophagy and CMA and from the availability of novel autophagic markers and better functional tests. Critical at this point is the understanding of how age-related changes in different hormonal and metabolic environments contribute to the diminished autophagic activity. The growing number of largescale proteomic and metabolomic-based studies on aging models should provide valuable clues on this matter.

Several studies analyzing autophagy in pathological conditions have concomitantly expanded our knowledge regarding the physiological roles of autophagy in multi-cellular organisms. However, a better understanding of the connections between autophagy and processes such as programmed cell death, malignant transformation, and tumor growth would be of particular interest. Pharmacological modulation of the disrupted autophagic process could be a very promising strategy for future therapies within a wide spectrum of pathological situations including cancer, neurological diseases, and premature aging.

\section{REFERENCES}

1. Goldberg AL, 2003 Protein degradation and protection against misfolded or damaged proteins. Nature 426:895899.

2. Ciechanover A, 2005 Proteolysis: from the lysosome to ubiquitin and the proteasome. Nat Rev Mol Cell Biol 6: 79-87.

3. Myung J, Kim KB, Crews CM, 2001 The ubiquitin-proteasome pathway and proteasome inhibitors. Med Res Rev 21: 245-273.

4. Sorimachi H, Ishiura S, Suzuki K, 1997 Structure and physiological function of calpains. Biochem J 328: 721732.

5. Cuervo AM, 2004 Autophagy: many paths to the same end. Mol Cell Biochem 263: 55-72.

6. Bakala H, Delaval E, Hamelin M, Bismuth J, Borot-Laloi C, Corman B, 2003 Changes in rat liver mitochondria with aging. Lon protease-like reactivity and $\mathrm{N}$ (epsilon)carboxymethyllysine accumulation in the matrix. Eur $\mathbf{J}$
Biochem 270: 2295-2302.

7. Arnold I, Langer T, 2002 Membrane protein degradation by AAA proteases in mitochondria. Biochim Biophys Acta 1592: 89-96.

8. Levine B, Klionsky DJ, 2004 Development by self-digestion: molecular mechanisms and biological functions of autophagy. Dev Cell 6: 463-477.

9. Lee HK, Marzella L, 1994 Regulation of intracellular protein degradation with special reference to lysosomes: role in cell physiology and pathology. Int Rev Exp Patho 35: 39-147.

10. Kabeya Y, Mizushima N, Ueno T, et al, 2000 LC3, a mammalian homologue of yeast Apg8p, is localized in autophagosome membranes after processing. EMBO J 19: 5720-5728.

11. De Duve C, Wattiaux R, 1966 Functions of lysosomes. Annu Rev Physiol 28: 435-492.

12. Reggiori F, Klionsky DJ, 2002 Autophagy in the eukaryotic cell. Eukaryot Cell 1: 11-21.

13. Wang CW, Klionsky DJ, 2003 The molecular mechanism of autophagy. Mol Med 9: 65-76.

14. Klionsky DJ, 2005 The molecular machinery of autophagy: unanswered questions. J Cell Sci 118: 7-18.

15. Dice J, 2000 Lysosomal pathways of protein degradation Austin: Landes Bioscience.

16. Dice JF, 2007 Chaperone-mediated autophagy. Autophagy 3: 295-299.

17. Majeski AE, Dice JF, 2004 Mechanisms of chaperonemediated autophagy. Int J Biochem Cell Biol 36: 24352444.

18. Dice JF, 1990 Peptide sequences that target cytosolic proteins for lysosomal proteolysis. Trends Biochem Sci 15: 305-309.

19. Hayes SA, Dice JF, 1996 Roles of molecular chaperones in protein degradation. Cell Biol 132: 255-258.

20. Cuervo AM, Dice JF, 1996 A receptor for the selective uptake and degradation of proteins by lysosomes. Science 273: 501-503.

21. Terlecky S, Chiang HL, Olson T, Dice J, 1992 Protein and peptide binding and stimulation of in vitro lysosomal proteolysis by the $73-\mathrm{KdA}$ heat shock cognate protein. $\mathrm{J}$ Biol Chem 267: 9202-9209.

22. Mortimore G, Lardeux BR, Adams CE, 1988 Regulation of microautophagy and basal protein turnover in rat liver. Effects of short-term starvation. J Biol Chem 263: 2506-2512.

23. Farre JC, Subramani S, 2004 Peroxisome turnover by micropexophagy: an autophagy-related process. Trends Cell Biol 14: 515-523.

24. Kim I, Rodriguez-Enriquez S, Lemasters JJ, 2007 Selective degradation of mitochondria by mitophagy. Arch Biochem Biophys 462: 245-253.

25. Teter SA, Klionsky DJ, 2000 Transport of proteins to the yeast vacuole: autophagy, cytoplasm-to-vacuole targeting, and role of the vacuole in degradation. Semin Cell Dev Biol 11: 173-179. 
26. Thumm M, 2000 Structure and function of the yeast vacuole and its role in autophagy. Microsc Res Tech 51: 563-572.

27. Harding TM, Hefner-Gravink M, Thumm M, Klionsky DJ, 1996 Genetic and phenotypic overlap between autophagy and the cytoplasm to vacuole protein targeting pathway. J Biol Chem 271: 17621-17624.

28. Klionsky DJ, Emr SD, 2000 Autophagy as a Regulated Pathway of Cellular Degradation. Science 290: 17171721.

29. Reggiori F, Klionsky DJ, 2005 Autophagosomes: biogenesis from scratch? Curr Opin Cell Biol 17: 415-422.

30. Shintani T, Klionsky DJ, 2004 Autophagy in health and disease: a double-edged sword. Science 306: 990-995.

31. Petiot A, Ogier-Denis E, Blommaart EF, Meijer AJ, Codogno P, 2000 Distinct classes of phosphatidylinositol 3'-kinases are involved in signaling pathways that control macroautophagy in HT-29 cells. J Biol Chem 275: 992998.

32. Schmelzle T, Hall MN, 2000 TOR, a central controller of cell growth. Cell 103: 253-262.

33. Kamada Y, Funakoshi T, Shintani T, Nagano K, Ohsumi M, Ohsumi Y, 2000 Tor-mediated induction of autophagy via an Apg1 protein kinase complex. J Cell Biol 150: 1507-1513.

34. Dunn WA Jr, 1990 Studies on the mechanisms of autophagy: formation of the autophagic vacuole. J Cell Biol 110: 1923-1933.

35. Seglen PO, Berg TO, Blankson H, Fengsrud M, Holen I, Stromhaug PE, 1996 Structural aspects of autophagy. Adv Exp Med Biol 389: 103-111.

36. Noda T, Suzuki K, Ohsumi Y, 2002 Yeast autophagosomes: de novo formation of a membrane structure. Trends Cell Biol 12: 231-235.

37. Kim J, Huang WP, Stromhaug PE, Klionsky DJ, 2002 Convergence of multiple autophagy and cytoplasm to vacuole targeting components to a perivacuolar membrane compartment prior to de novo vesicle formation. J Biol Chem 277: 763-773.

38. Ohsumi Y, 2001 Molecular dissection of autophagy: two ubiquitin-like systems. Nat Rev Mol Cell Biol 2: 211-216.

39. Suzuki K, Ohsumi Y, 2007 Molecular machinery of autophagosome formation in yeast, Saccharomyces cerevisiae. FEBS Lett 581: 2156-2161.

40. Takeshige K, Baba M, Tsuboi S, et al, 1992 Autophagy in yeast demonstrated with Proteinase-deficient mutants and conditions for its induction. Cell Biol 119: 301-311.

41. Thumm M, Egner R, Koch B, et al, 1994 Isolation of autophagocytosis mutants of Saccharomyces cerevisiae. FEBS Lett 349: 275-280.

42. Klionsky DJ, Cregg JM, Dunn WA Jr, et al, 2003 A unified nomenclature for yeast autophagy-related genes. Dev Cell 5: 539-545.

43. Meijer AJ, Codogno P, 2004 Regulation and role of autophagy in mammalian cells. Int $\mathrm{J}$ Biochem Cell Biol
36: 2445-2462.

44. Meijer AJ, Codogno P, 2006 Signalling and autophagy regulation in health, aging and disease. Mol Aspects Med 27: 411-425.

45. Beck T, Hall MN, 1999 The TOR signalling pathway controls nuclear localization of nutrient-regulated transcription factors. Nature 402: 689-692.

46. Holen I, Gordon PB, Seglen PO, 1992 Protein kinase dependent effects of okadaic acid on hepatocytic autophagy and cytoskeletal integrity. Biochem J 284: 633-636.

47. Mavrakis M, Lippincott-Schwartz J, Stratakis CA, Bossis I, 2006 Depletion of type IA regulatory subunit (RIalpha) of protein kinase A (PKA) in mammalian cells and tissues activates mTOR and causes autophagic deficiency. Hum Mol Genet 15: 2962-2971.

48. Mavrakis M, Lippincott-Schwartz J, Stratakis CA, Bossis I, 2007 mTOR kinase and the regulatory subunit of protein kinase A (PRKAR1A) spatially and functionally interact during autophagosome maturation. Autophagy 3: 151-153.

49. Kamada Y, Funakoshi T, Shintani T, Nagano K, Ohsumi M, Ohsumi Y, 2000 Tor-mediated induction of autophagy via an Apg1 protein kinase complex. J Cell Biol 150: 1507-1513.

50. Okazaki N, Yan J, Yuasa S, et al, 2000 Interaction of the Unc-51-like kinase and microtubule-associated protein light chain 3 related proteins in the brain: possible role of vesicular transport in axonal elongation. Brain Res Mol Brain Res 85: 1-12.

51. Yan J, Kuroyanagi H, Kuroiwa A, et al, 1998 Identification of mouse ULK1, a novel protein kinase structurally related to C. elegans UNC-51. Biochem Biophys Res Commun 246: 222-227.

52. Seglen PO, Gordon PB, 1982 3-Methyladenine: specific inhibitor of autophagic/lysosomal protein degradation in isolated rat hepatocytes. Proc Natl Acad Sci 79: 18891892.

53. Kihara A, Noda T, Ishihara N, Ohsumi Y, 2001 Two distinct Vps34 phosphatidylinositol 3-kinase complexes function in autophagy and carboxypeptidase Y sorting in Saccharomyces cerevisiae. J Cell Biol 152: 519-530.

54. Petiot A, Ogier-Denis E, Blommaart EF, Meijer AJ, Codogno P, 2000 Distinct classes of phosphatidylinositol 3'-kinases are involved in signaling pathways that control macroautophagy in HT-29 cells. J Biol Chem 275: 992998.

55. Liang XH, Jackson S, Seaman M, et al, 1999Induction of autophagy and inhibition of tumorigenesis by beclin 1. Nature 402: 672-676.

56. Harman D, 2001 Aging: overview. Ann N Y Acad Sci 928: 1-21.

57. Sohal RS, Ku HH, Agarwal S, Forster MJ, Lal H, 1994 Oxidative damage, mitochondrial oxidant generation and antioxidant defenses during aging and in response to food restriction in the mouse. Mech Ageing Dev 74: 121-133. 
58. Weindruch R, Sohal RS, 1997 Seminars in medicine of the Beth Israel Deaconess Medical Center. Caloric intake and aging. N Engl J Med 337: 986-994.

59. Terman A, 2001 Garbage catastrophe theory of aging: imperfect removal of oxidative damage? Redox Rep 6: 15-26.

60. Weinert BT, Timiras PS, 2003 Invited review: Theories of aging. J Appl Physiol 95: 1706-1716.

61. Harman D, 1956 Aging: a theory based on free radical and radiation chemistry. J Gerontol 11: 298-300.

62. Beckman KB, Ames BN, 1998 The free radical theory of aging matures. Physiol Rev 78:547-581.

63. Miquel J, Economos AC, Fleming J, Johnson Jr. JE, 1980 Mitochondrial role in cell aging. Exp Gerontol 15: 579-591.

64. Halliwell B, Gutteridge JMC, 1999 Free Radicals in Biology and Medicine, 3rd ed Oxford University Press: New York.

65. Ozawa T, 1997 Genetic and functional changes in mitochondria associated with aging. Physiol Rev 77: 425464.

66. Khrapko K, Bodyak N, Thilly WG, et al,1999 Cell-bycell scanning of whole mitochondrial genomes in aged human heart reveals a significant fraction of myocytes with clonally expanded deletions. Nucleic Acids Res 27: 2434-2441.

67. Taylor RW, Barron MJ, Borthwick GM, et al, 2003 Mitochondrial DNA mutations in human colonic crypt stem cells. J Clin Invest 112: 1351-1360.

68. Hochhauser D, 2000 Relevance of mitochondrial DNA in cancer. Lancet 356: 181-182.

69. Trifunovic A, Wredenberg A, Falkenberg M, et al, 2004 Premature ageing in mice expressing defective mitochondrial DNA polymerase. Nature 429: 417-423.

70. Ermini M, 1976 Ageing changes in mammalian skeletal muscle: biochemical studies. Gerontology 22: 301-316.

71. Rodriguez-Enriquez S, He L, Lemasters JJ, 2004 Role of mitochondrial permeability transition pores in mitochondrial autophagy. Int J Biochem Cell Biol 36: 24632472.

72. Terman A, Brunk UT, 2005 Autophagy in cardiac myocyte homeostasis, aging, and pathology. Cardiovasc Res 68: 355-365.

73. Shigenaga MK, Hagen TM, Ames BN, 1994 Oxidative damage and mitochondrial decay in aging. Proc Natl Acad Sci 91: 10771-10778.

74. Wei YH, Lee HC, 2007 Oxidative stress, mitochondrial DNA mutation, and impairment of antioxidant enzymes in aging. Exp Biol Med (Maywood) 227: 671-682.

75. Perichon R, Bourre JM, Kelly JF, Roth GS, 1998 The role of peroxisomes in Aging. Cell Mol Life Sci 54: 641652.

76. Zs-Nagy I, 1994 The Membrane Hypothesis of Aging, CRC Press, Boca Raton, USA.

77. Matsuo M, Gomi F, Kuramoto K, Sagai M, 1993 Food restriction suppresses an age-dependent increase in the exhalation rate of pentane from rats: a longitudinal study. J Gerontol 48: B133-B136.

78. Dolfi C, Bergamini E, Carresi C, et al, 2003 The age-related accumulation of dolichol in rat liver may correlate with expectation of life. Biogerontology 4: 113-118.

79. Marino M, Dolfi C, Paradiso C, et al, 1998 Age-dependent accumulation of dolichol in rat liver: is tissue dolichol a biomarker of aging? J Gerontol A Biol Sci Med Sci 53: B87-B93.

80. Cerami A, 1985 Hypothesis. Glucose as a mediator of aging. J Am Geriatr Soc 33: 626-634.

81. Bonnefont-Rousselot D, Bastard JP, Jaudon MC, Delattre J, 2000 Consequences of the diabetic status on the oxidant/antioxidant balance. Diabetes Metab 26: 163-176.

82. Robins SP, 2007, Biochemistry and functional significance of collagen cross-linking. Biochem Soc Trans 35(Pt 5): 849-852.

83. Kumar PA, Kumar MS, Reddy GB, 2007 Effect of glycation on alpha-crystallin structure and chaperone-like function. Biochem J 408: 251-258.

84. Peppa M, Vlassara H, 2005 Advanced glycation end products and diabetic complications: A General overview. Hormones (Athens) 4: 28-37.

85. Jean-Pierre Tricot, 2006 Nobel prize winner Christian de Duve. From insulin to lysosomes. Hormones (Athens) 5: 151-155.

86. Dice J, 2000 Lysosomal pathways of protein degradation. Austin: Landes Bioscience, p 108.

87. Martinez-Vicente M, Sovak G, Cuervo AM, 2005 Protein degradation and aging. Exp Gerontol 40: 622-633.

88. Shibatani T, Ward W, 1996 Effect of age and food restriction on alkaline protease activity in rat liver. J Gerontol 51: B316-B322.

89. Glaser T, Schwarz-Ben Meir N, Barnoy S, Barak S, Eshhar Z, Kosower N, 1994 Calpain (Ca+2-dependent thiol protease) in erythrocytes of young and old individuals. Proc Natl Acad Sci 91: 7879-7883.

90. Brunk UT, Terman A, 2002 Lipofuscin: mechanisms of age-related accumulation and influence on cell functions. Free Radic Biol Med 33: 611-619.

91. Terman A, Dalen H, Brunk UT,1999 Ceroid/lipofuscinloaded human fibroblasts show decreased survival time and diminished autophagocytosis during amino acid starvation. Exp Gerontol 34: 943-957.

92. Terman A, Gustafsson B, Brunk UT, 2007 Autophagy, organelles and ageing. J Pathol 211: 134-143.

93. Terman A, Brunk UT, 1998 Ceroid/lipofuscin formation in cultured human fibroblasts: the role of oxidative stress and lysosomal proteolysis. Mech Ageing Dev 104: 277291.

94. Nakano M, Oenzil F, Mizuno T, Gotoh S, 1995 Agerelated changes in the lipofuscin accumulation of brain and heart. Gerontology 41: 69-79.

95. Ku HH, Brunk UT, Sohal RS, 1993 Relationship between mitochondrial superoxide and hydrogen peroxide production and longevity of mammalian species. Free Radic 
Biol Med 15: 621-627.

96. Cuervo AM, Dice JF, 2000 Age related decline in chaperone mediated autophagy. J Biol Chem 275: 3150531513.

97. Nishino I, 2003 Autophagic vacuolar myopathies. Curr Neurol Neurosci Rep 3: 64-69.

98. Nishino I, Fu J, Tanji K, et al, 2000 Primary LAMP-2 deficiency causes $\mathrm{X}$-linked vacuolar cardiomyopathy and myopathy (Danon disease). Nature 406: 906-910.

99. Kalimo H, Savontaus ML, Lang H, et al, 1988 X-linked myopathy with excessive autophagy: a new hereditary muscle disease. Ann Neurol 23: 258-265.

100. Nonaka I, 1999 Distal myopathies. Curr Opin Neurol 12: 493-499.

101. Goto Y, Komiyama A, Tanabe Y, Katafuchi Y, Ohtaki E, Nonaka I 1990 Myopathy in Marinesco- Sjpgren syndrome: an ultrastructural study. Acta Neuropathol (Berl) 80: 123-128.

102. Anglade P, Vyas S, Javoy-Agid F, et al, 1997 Apoptosis and autophagy in nigral neurons of patients with Parkinson's disease. Histol Histopathol 12: 25-31.

103. Cataldo AM, Hamilton DJ, Barnett JL, Paskevich PA, Nixon RA, et al, 1996 Properties of the endosomal-lysosomal system in the human central nervous system: disturbances mark most neurons in populations at risk to degenerate in Alzheimer's disease. J Neurosci 16: 186-199.

104. Kegel KB, Kim M, Sapp E, et al, 2000 Huntingtin expression stimulates endosomal-lysosomal activity, endosome tubulation, and autophagy. J Neurosci 20: 7268-7278.

105. Liberski PP, Sikorska B, Bratosiewicz-Wasik J, Gajdusek DC, Brown P, 2004 Neuronal cell death in transmissible spongiform encephalopathies (prion diseases) revisited: from apoptosis to autophagy. Int J Biochem Cell Biol 36: 2473-2490.

106. Stefanis L, Larsen KE, Rideout HJ, Sulzer D, Greene LA, 2001 Expression of A53T mutant but not wild-type alpha-synuclein in PC12 cells induces alterations of the ubiquitin-dependent degradation system, loss of dopamine release, and autophagic cell death. J Neurosci 21: 9549-9560.

107. Venkatraman P, Wetzel R, Tanaka M, Nukina N, Goldberg AL, 2004 Eukaryotic proteasomes cannot digest polyglutamine sequences and release them during degradation of polyglutamine-containing proteins. Mol Cell 14: 95-104.

108. Teckman, JH, An JK, Blomenkamp K, Schmidt B, Perlmutter D, 2004 Mitochondrial autophagy and injury in the liver in alpha 1-antitrypsin deficiency. Am J Physiol Gastrointest Liver Physiol 286: 851-862.

109. Dorn BR, Dunn WA Jr, Progulske-Fox A, 2002 Bacterial interactions with the autophagic pathway. Cell Microbiol 4: $1-10$.

110. Coers J, Kagan JC, Matthews M, Nagai H, Zuckman DM, Roy CR, 2000 Identification of Icm protein complexes that play distinct roles in the biogenesis of an organelle permissive for Legionella pneumophila intracellular growth. Mol Microbiol 38: 719-736.

111. Talloczy Z, Jiang W, Virgin HW 4th, et al, 2002 Regulation of starvation- and virus-induced autophagy by the eIF2 alpha kinase signaling pathway. Proc Natl Acad Sci 99: 190-195.

112. Bergamini E, Cavallini G, Donati A, Gori Z, 2004 The role of macroautophagy in the ageing process, anti-ageing intervention and age-associated diseases. Int J Biochem Cell Biol 36: 2392-2404.

113. Guertin DA, Sabatini DM, 2005 An expanding role for mTOR in cancer. Trends Mol Med 11: 353-361.

114. Komatsu M, Waguri S, Chiba T, et al, 2006 Loss of autophagy in the central nervous system causes neurodegeneration in mice. Nature 441: 880-884.

115. Stefanis L, 2005 Caspase-dependent and -independent neuronal death: two distinct pathways to neuronal injury. Neuroscientist 11: 50-62.

116. Webb JL, Ravikumar B, Atkins J, Skepper JN, Rubinsztein DC, 2003 Alpha-Synuclein is degraded by both autophagy and the proteasome. J Biol Chem 278: 25009-25013.

117. Cuervo AM, Stefanis L, Fredenburg R, Lansbury PT, Sulzer D, 2004 Impaired degradation of mutant alphasynuclein by chaperone-mediated autophagy. Science 305: 1292-1295.

118. Yu WH, Cuervo AM, Kumar A, et al, 2005 Macroautophagy--a novel Beta-amyloid peptide-generating pathway activated in Alzheimer's disease. J Cell Biol 171: 87-98.

119. Nixon R, Cataldo A, 2006 Lysosomal disease pathways: genes to neurodegeneration in Alzheimer's disease. $\mathrm{J}$ Alzheimers Dis 9: 277-289.

120. Nixon R, Wegiel J, Kumar A, et al, 2005 Extensive involvement of autophagy in Alzheimer disease: an immuno-electron microscopy study. J Neuropathol Exp Neurol 64: 113-122.

121. Glabe C, 2001 Intracellular mechanisms of amyloid accumulation and pathogenesis in Alzheimer's disease. $\mathrm{J}$ Mol Neurosci 17: 137-145.

122. Cataldo AM, Peterhoff CM, Schmidt SD, et al, 2004 Presenilin mutations in familial Alzheimer disease and transgenic mouse models accelerate neuronal lysosomal pathology. J Neuropathol Exp Neurol 63: 821-830.

123. Wilson CA, Murphy DD, Giasson Bi, Zhang B, Trojanowski JO, Lee VM, 2004 Degradative organelles containing mislocalized alpha-and beta-synuclein proliferate in presenilin-1 null neurons. J Cell Biol 165: 335-346.

124. Landles C, Bates GP, 2004 Huntingtin and the molecular pathogenesis of Huntington's disease. Fourth in molecular medicine review series. EMBO Rep 5: 958-963.

125. Ravikumar B, Duden R, Rubinsztein DC, 2002 Aggregate-prone proteins with polyglutamine and polyalanine expansions are degraded by autophagy. Hum Mol Genet 11: $1107-1117$.

126. Sapp E, Schwarz C, Chase K, et al, 1997 Huntingtin localization in brains of normal and Huntington's disease 
patients. Ann Neurol 42: 604-612.

127. Nagata E, Sawa A, Ross CA, Snyder SH, 2004 Autophagosome-like vacuole formation in Huntington's disease lymphoblasts. NeuroReport 15: 1325-1328.

128. Kegel KB, Kim M, Sapp E, et al, 200 Huntingtin expression stimulates endosomal-lysosomal activity, endosome tubulation, and autophagy. J Neurosci 20: 7268-7278.

129. Ravikumar B, Acevedo-Arozena A, Imarisio S, et al, 2005 Dynein mutations impair autophagic clearance of aggregate-prone proteins. Nat Genet 37: 771-776.

130. Ravikumar B, Vacher C, Berger Z, et al, 2004 Inhibition of mTOR induces autophagy and reduces toxicity of polyglutamine expansions in fly and mouse models of Huntington disease. Nat Genet 36: 585-595.

131. Hickey MA, Chesselet MF, 2003 Apoptosis in Huntington's disease. Prog Neuropsychopharmacol Biol Psychiatry 27: 255-265.

132. Martin DN, Baehrecke EH, 2004 Caspases function in autophagic programmed cell death in Drosophila. Development 131: 275-284.
133. Mizushima N, Yamamoto A, Matsui M, Yoshimori T, Ohsumi Y, 2004 In vivo analysis of autophagy in response to nutrient starvation using transgenic mice expressing a fluorescent autophagosome marker. Mol Biol Cell 15: 1101-1111.

134. Brunk UT, Terman A, 2002 The mitochondrial-lysosomal axis theory of aging: accumulation of damaged mitochondria as a result of imperfect autophagocytosis. Eur J Biochem 269: 1996-2002.

135. Del Roso A, Vittorini S, Cavallini G, et al, 2003 Ageing-related changes in the in vivo function of rat liver macroautophagy and proteolysis. Exp Gerontol 38: 519527.

136. Melendez A, Talloczy Z, Seaman M, Eskelinen EL, Hall DH, Levine B, 2003 Autophagy genes are essential for dauer development and life-span extension in C. elegans. Science 301: 1387-1391.

137. Vellai T, Takacs-Vellai K, Zhang Y, Kovacs AL, Orosz L, Muller F, 2003 Influence of TOR kinase on lifespan in C. elegans. Nature 426: 620. 